\title{
Neutrino Cooling of Primordial Hot Regions
}

\author{
Konstantin Belotsky ${ }^{1, *}$, Mohamed El Kasmi ${ }^{1,2}$ and Sergey Rubin ${ }^{1,3}$ \\ 1 National Research Nuclear University MEPhI (Moscow Engineering Physics Institute), Kashirskoe Shosse 31, \\ 115409 Moscow, Russia; m.elkasemy@science.sohag.edu.eg (M.E.K.); sgrubin@mephi.ru (S.R.) \\ 2 Physics Department, Faculty of Science, Sohag Center, Sohag University, Sohag 82524, Egypt \\ 3 N. I. Lobachevsky Institute of Mathematics and Mechanics, Kazan Federal University, \\ Kremlevskaya Street 18, 420008 Kazan, Russia \\ * Correspondence: kmbelotskij@mephi.ru
}

Received: 7 August 2020; Accepted: 28 August 2020; Published: 1 September 2020

\begin{abstract}
The effect of neutrino cooling of possible primary regions filled by hot matter is discussed. Such regions could be obtained from the primordial density inhomogeneities and survive up to the modern epoch. The inhomogeneities could be caused by a symmetry breaking during the inflationary stage. We show that the final temperature of such region should be $\sim 10 \mathrm{keV}$ provided that the initial temperature is within the interval $10 \mathrm{keV} \div 100 \mathrm{MeV}$. The cooling is realized due to the weak nuclear reactions containing $n-p$ transition. The lower limit $10 \mathrm{keV}$ is accounted for by suppression of the reactions rates because of the threshold effect and particle concentration decrease.
\end{abstract}

Keywords: primordial hot regions; neutrino cooling; primordial black holes

\section{Introduction}

The evolution of the early universe is one of the main testbeds for a study of both elementary particle physics models and cosmological models. Any imprints of the physics at early stage and phenomena which are responsible for them, deserves careful analysis.

In this paper, we discuss local heated areas that could appear in the early universe. There are some observations [1] favouring their creation during the early expansion. Similar local heated areas have been hypothetically discussed earlier [2-4]. They could separate from the Hubble flow if their density is high enough. For instance, such regions could be formed by clusters of primordial black holes (PBH) $[1,5]$. The latter could arise as the result of the symmetry breaking by the inflaton fluctuations near a maximum of its potential [6-8]. In the following, origin of the regions is not specified.

We also assume that the matter including the dark matter was captured by gravitational forces of the area even before formation of stars, when the temperature of the universe media was high enough. There are several reasons for an unusual temperature evolution within such regions. Some of them are discussed below.

Diffusive character of particle propagation at the temperature $T \lesssim 1 \mathrm{MeV}$ prevents expansion of heat out of the region [2]. The escape time of electrons from the region of the size $R$ with temperature $T \sim m_{e}$ would be [9]

$$
t \sim \frac{R^{2}}{D} \sim R^{2} T \alpha^{2} \sim 10^{24} \text { years. }
$$

To obtain a numerical estimate we suppose that $R \sim 1 \mathrm{pc}$ as has been obtained in [5], the electron velocity $v$ and the Coulomb logarithm $L$ were supposed to be of order 1 , diffusion coefficient was estimated from the electron travelling length [9] $D \sim \lambda v \sim T^{2} / \alpha^{2} n$ with $n \sim T^{3}$ being the electron concentration. One may then see that the diffusion time is much bigger than the universe lifetime for $R \ll 1 \mathrm{pc}$. The situation with the neutrino is different. Because of its finite size, the region can be transparent for neutrinos even at $T>1 \mathrm{MeV}$. 
The initial mass of the region can be as large as the mass of matter inside horizon at the temperature $T_{h}$ when the region started to form, i.e., roughly $M \sim 10^{6}\left(\mathrm{MeV} / T_{h}\right)^{2} M_{\odot}$. Consequently, $T_{h}>10-100 \mathrm{MeV}$ is of less interest due to the low mass of the region. Nevertheless, the effects considered here should be applicable to small regions as well.

After virialization (at some temperature $T_{0}<T_{h}$, referred hereafter to as initial temperature), the regions can be heated or cooled due to a variety of processes inside them. Among them are neutrino cooling, nuclear reactions, radiation of the hot plasma and the stars formed inside the region [10], gravitational dynamics of the system, shock waves, diffusion of matter, variation of the vacuum state while the region is born [11], energy transfer from collapsing walls [12-15], accretion, Hawking evaporation. The last mechanisms are only present if PBHs have been created [16,17]. Neutrino cooling could be the most efficient mechanism among those listed in a certain energy range.

Neutrino cooling is realized due to reactions of weak $p \leftrightarrow n$ transitions and $e^{ \pm}$annihilation. The reaction rate slows down with cooling of the region due to threshold effects and a decrease in the concentrations $n$ and $e^{ \pm}$. Here we show that the temperature tends to a fixed value.

\section{Reactions Inside Heated Area}

The size, mass and temperature of a volume separated from the cosmological expansion depend on many factors. When choosing the initial conditions, we will partially follow the paper [5] where the mass of the initially trapped matter is varied in wide range $10^{4}-10^{8} \mathrm{M}_{\odot}$. The main initial parameters describing the hot area are assumed as follows: the size of the region is about $1 \mathrm{pc}$, its mass $10^{4} M_{\odot}$, initial temperature is in the interval $T_{0} \sim 1 \mathrm{keV} \div 10 \mathrm{MeV}$ (at higher temperature cluster is not transparent for the neutrino). The change of the parameters in wide range does not affect the qualitative effect.

Consider the cooling of the heated area due to the neutrino radiation. The basic reactions of the neutrino production are the following

$$
\begin{gathered}
e^{-}+p \rightarrow n+v_{e}, \\
e^{+}+n \rightarrow p+\bar{v}_{e}, \\
e^{+}+e^{-} \rightarrow v_{e, \mu, \tau}+\bar{v}_{e, \mu, \tau}, \\
n \rightarrow p+e^{-}+\bar{v}_{e} .
\end{gathered}
$$

The neutrino production rate per unit volume, $\gamma_{i} \equiv \Gamma_{i} / V$, are respectively

$$
\begin{array}{r}
\gamma_{e p}=n_{e^{-}} n_{p} \sigma_{e p} v, \\
\gamma_{e n}=n_{e^{+}} n_{n} \sigma_{e n} v, \\
\gamma_{e e}=n_{e^{-}} n_{e^{+}} \sigma_{e e} v, \\
\gamma_{n}=\frac{n_{n}}{\tau_{n}}
\end{array}
$$

for each reaction. Here $n_{i}$ is the concentration of the respective species, $\sigma_{i j}$ is the cross section of interacting particles $i$ and $j, v$ is their relative velocity, which is supposed to be equal 1 for all the reactions and $\tau_{n} \approx 1000 \mathrm{~s}$ is the neutron lifetime.

The number densities are approximately described by the following formulas

$$
\begin{gathered}
n_{p}=\frac{n_{B}}{1+\exp \left(-\frac{\Delta m}{T}\right)}, \quad n_{n}=n_{p}(T) \exp \left(-\frac{\Delta m}{T}\right), \\
n_{e^{-}}=n_{e}^{e q}(T)+\Delta n_{e}, \quad n_{e^{+}}=n_{e}^{e q}(T) \exp \left(-\frac{m_{e}}{T}\right), \\
n_{B} \equiv n_{p}+n_{n}=g_{B} \eta n_{\gamma}\left(T_{0}\right), \quad \Delta n_{e} \equiv n_{e^{-}}-n_{e^{+}}=n_{p} .
\end{gathered}
$$


Here $\eta=n_{B} / n_{\gamma} \approx 0.6 \cdot 10^{-9}$ is the baryon to photon relation in the modern universe, $g_{B} \sim 1$ is the correction factors of that relation due to entropy re-distribution, $n_{\gamma}(T)=\frac{2 \zeta(3)}{\pi^{2}} T^{3}$ and $n_{e}^{e q}=$ $\frac{3 \zeta(3)}{2 \pi^{2}} T^{3} \approx 9 \cdot 10^{25}\left(\frac{T}{100 \mathrm{keV}}\right)$ are the equilibrium photon and electron number densities respectively, $\Delta m=m_{n}-m_{p}=1.2 \mathrm{MeV}$. One should pay attention that $n_{\gamma}$ depends on $T_{0}$ rather than $T$. We consider all densities to be independent on space coordinate within the region. The view of formula Equation (10) was assumed from consideration of due asymptotics of $n_{e^{ \pm}}(T)$ at $T \gg m_{e}$ and $T \ll m_{e}$. We checked that variation of this view does not make difference of principle in the result.

The following approximate formulas

$$
\begin{array}{r}
\sigma_{e n}=\sigma_{e e}=\sigma_{w}, \\
\sigma_{e p}=\sigma_{w} \exp \left(-\frac{Q}{T}\right),
\end{array}
$$

are used for the cross sections (exacter for their thermally averaged values $\left\langle\sigma_{i} v\right\rangle$ ). Here $\sigma_{w} \sim G_{\mathrm{F}}^{2} T^{2}$, $Q=m_{n}-\left(m_{e}+m_{p}\right)=0.77 \mathrm{MeV}$ effectively takes into account the threshold effect in respective reaction, $G_{\mathrm{F}}=1.166 \cdot 10^{-5} \mathrm{GeV}^{-2}$ is the Fermi constant. Such an estimation of cross section has accuracy factor 3 of in the range where it is relevant.

The backward reactions for Equations (1)-(4) are suppressed since neutrino freely escape the cluster. This is essential feature of the considered case distinguishing it from the processes in the early universe. Indeed, the neutrino would scatter off $e^{ \pm}$, with the mean free path $\lambda_{v}=1 / n_{e} \sigma_{v e} \simeq 10^{24} \mathrm{~cm}$ at the temperature $T=100 \mathrm{keV}$. Hence, $\lambda_{v}$ of the neutrino inside the cluster would be much larger than its size, 1 pc in our case. This justifies the importance of study the energy outflow by neutrinos and subsequent cooling of the media inside the cluster.

Notice that the densities of the relativistic matter components like $e^{ \pm}$and $\gamma$ are functions of the current temperature $T$ within the region (which is varied due to neutrino cooling and possible other effects), contrarily to the baryon density. The latter is defined by initial temperature $T_{0}$ and almost unchanged with time.

\section{Temperature Evolution}

The temperature balance

$$
\Delta Q=\delta U
$$

follows from the first law of thermodynamics. Here $\Delta Q$ and $\delta U$ are the heat outflow due to neutrinos and the diminution of inner energy of the matter inside the cluster respectively. Applying it to our case, we obtain the energy conservation in the form

$$
-\left(\gamma_{e n}+\gamma_{e p}+\gamma_{e e}+\gamma_{n}\right) E_{v} d t=4 b T^{3} d T
$$

where $E_{v} \sim T$ is the energy of outgoing neutrino, $b=\pi^{2} / 15$ is the radiation constant.

Integrating Equation (15), one can get time dependence of the temperature in an implicit form

$$
\Delta t=-4 b \int_{T_{0}}^{T} \frac{T^{\prime 2} d T^{\prime}}{\gamma_{e n}+\gamma_{e p}+\gamma_{e e}+\gamma_{n}} .
$$

Here explicit dependence of the functions $\gamma_{i}$ on $T$ is the following: $\gamma_{e n}=C_{1} \cdot T^{5} \exp \left(-\frac{Q}{T}\right)$, $\gamma_{e p}=C_{2} \cdot T^{5} \exp \left(-\frac{m_{e}+\Delta m}{T}\right), \gamma_{e e}=C_{3} \cdot T^{8} \exp \left(-\frac{m_{e}}{T}\right), \gamma_{n}=C_{4} \cdot \exp \left(-\frac{\Delta m}{T}\right)$, where exact view of $C_{i}$ follows from Equations (5)-(13) and their specifications in the text. The coefficients $C_{1,2,4}$ also contain the multiplier $\left[1+\exp \left(-\frac{\Delta m}{T}\right)\right]^{-1}$.

Numerical solutions of Equation (16) are represented in Figure 1 left panel, where different curves correspond to different initial temperatures $T_{0}$. As seen, the temperature falls sharply around 
$T \sim 100 \mathrm{keV}$ and then smoothly subsides to $\sim 10 \mathrm{keV}$ during a period $t_{\text {cooling. }}$. It is drawn in Figure 1 right.
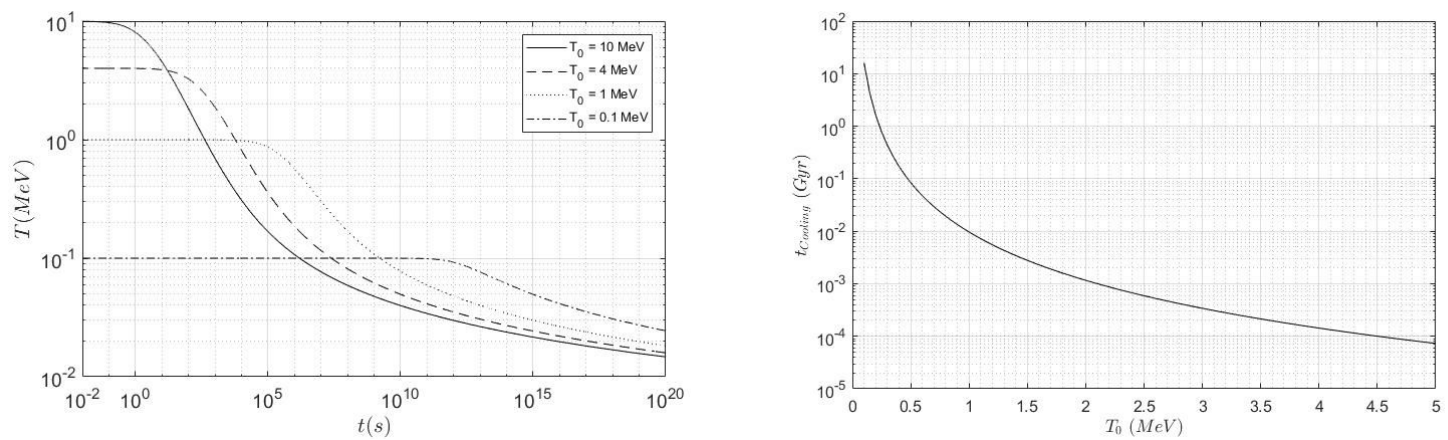

Figure 1. (Left) The time behaviour of the temperature inside the heated area. (Right) Cooling time $t_{\text {cooling }}$ of media inside the heated area depending on the initial temperature $T_{0}$.

As one can see, neutrino cooling effect can be essential for the heated areas with initial temperature $T_{0} \gtrsim 100 \mathrm{keV}$.

Behaviour for different initial temperature $T_{0}$ in Figure 1, left can be qualitatively explained. The higher initial temperature is, the higher initial densities of all matter components are. At the same time, the baryon density does not change with time in the gravitationally separated regions and $\Delta n_{e}$ changes slowly. Hence, the nuclear reactions proceed faster for higher $T_{0}$. This speculation is confirmed by the curves represented in Figure 1, left panel.

\section{Conclusions}

Relic hot regions are widely discussed in the literature, some of them are mentioned in the Introduction. Their existence could open new ways for research and study observational effects.

In this letter, we study neutrino mechanism of cooling of such regions. We have shown that these regions are cooling down to the temperature value $\sim 0.01 \div 0.1 \mathrm{MeV}$ due to neutrino emission. In the course of successive evolution up to the present times, the temperature varies slowly.

There are other heating and cooling effects that seem less decisive than the neutrino emission process. Nevertheless, their study should be carried out in the future for more accurate description of evolution of such regions. It is worth mentioning a variety of the nuclear reactions, growth of the region due to the pressure excess and so on.

It is worth paying attention to $\mathrm{PBH}$ as a possible origin of such regions. This is unique phenomena indicating possible deviation from the standard astrophysical scenario (see, e.g., recent works [18-22]). They are often involved in attempts to unravel a variety of the cosmological puzzles.

PBH cluster could naturally account for existence of such regions [1,5]. Moreover, the domain-wall mechanism of the cluster formation $[8,14,23]$ can lead to additional heating of the matter inside it [11-14]. Further analysis of observational effects of such regions, as well as point-like cosmic gamma-ray sources [24] and gravitation lensing events [25], would provide an important test of the hypothesis on the PBH cluster existence.

Author Contributions: Conceptualization, S.R. and K.B.; methodology, S.R. and K.B.; software and calculation, M.E.K.; validation, S.R., K.B. and M.E.K.; formal analysis; investigation; resources; data curation; writing-original draft preparation, S.R., K.B. and M.E.K.; writing-review and editing, S.R., K.B. and M.E.K.; visualization; supervision, S.R. and K.B.; project administration; funding acquisition, S.R. and K.B. All authors have read and agreed to the published version of the manuscript.

Funding: The work was supported by the Ministry of Science and Higher Education of the Russian Federation as part of the Program for Improving the Competitiveness of the MEPhI (project no. 02. a03. 21.0005), and also (work of K.B.) by project No 0723-2020-0040 "Fundamental problems of cosmic rays and dark matter". The work of S.R. 
is supported by the RFBR grant 19-02-00930 and is performed according to the Russian Government Program of Competitive Growth of Kazan Federal University.

Acknowledgments: Our special thanks to M. Khlopov for useful discussion. We would like to thank A. Kirillov, $\mathrm{V}$. Nikulin for the interest and help in some issues.

Conflicts of Interest: The authors declare no conflict of interest.

\section{References}

1. Kashlinsky, A.; Ali-Haïmoud, Y.; Clesse, S.; Garcia-Bellido, J.; Amendola, L.; Wyrzykowski, L.; Annis, J.; Arbey, A.; Arendt, R.; Atrio-Barandela, F.; et al. Electromagnetic probes of primordial black holes as dark matter. Bull. Am. Astron. Soc. 2019, 51, 51.

2. Dubrovich, V.K.; Glazyrin, S.I. Cosmological dinosaurs. arXiv 2012, arXiv:1208.3999.

3. Kumar, S.; Dimastrogiovanni, E.; Starkman, G.D.; Copi, C.; Lynn, B. CMB spectral distortions from cooling macroscopic dark matter. Phys. Rev. D 2019, 99. [CrossRef]

4. Kogut, A.; Abitbol, M.; Chluba, J.; Delabrouille, J.; Fixsen, D.; Hill, J.; Patil, S.; Rotti, A. CMB Spectral Distortions: Status and Prospects. arXiv 2019, arXiv:1907.13195.

5. Belotsky, K.M.; Dokuchaev, V.I.; Eroshenko, Y.N.; Esipova, E.A.; Khlopov, M.Y.; Khromykh, L.A.; Kirillov, A.A.; Nikulin, V.V.; Rubin, S.G.; Svadkovsky, I.V. Clusters of Primordial Black Holes. Eur. Phys. J. C 2019, 79. [CrossRef]

6. Gani, V.A.; Kirillov, A.A.; Rubin, S.G. Classical transitions with the topological number changing in the early Universe. J. Cosmol. Astropart. Phys. 2018, 4, 042. [CrossRef]

7. Dokuchaev, V.; Eroshenko, Y.; Rubin, S. Early formation of galaxies initiated by clusters of primordial black holes. Astron. Rep. 2008, 52, 779-789. [CrossRef]

8. Rubin, S.G.; Sakharov, A.S.; Khlopov, M.Y. The Formation of primary galactic nuclei during phase transitions in the early universe. J. Exp. Theor. Phys. 2001, 92, 921-929. [CrossRef]

9. Lifshitz, E.; Pitaevskii, L. Physical Kinetics. Course of Theoretical Physics; Oxford University Press, Oxford, UK, 2002.

10. Dolgov, A.; Postnov, K. Electromagnetic radiation accompanying gravitational waves from black hole binaries. J. Cosmol. Astropart. Phys. 2017, 2017, 018. [CrossRef]

11. Belotsky, K.; Golikova, Y.; Rubin, S. Local heating of matter in the early universe owing to the interaction of the Higgs field with a scalar field. Phys. Atom. Nucl. 2017, 80, 718-720. [CrossRef]

12. Berezin, V.; Kuzmin, V.; Tkachev, I. Thin-wall vacuum domain evolution. Phys. Lett. B 1983, 120, 91-96. [CrossRef]

13. Khlopov, M.; Konoplich, R.; Rubin, S.; Sakharov, A. Formation of black holes in first order phase transitions. arXiv 1998, arXiv:hep-ph/9807343.

14. Rubin, S.G.; Khlopov, M.Y.; Sakharov, A.S. Primordial Black Holes from Non-Equilibrium Second Order Phase Transition. Gravit. Cosmol. 2000, 6, 51-58.

15. Deng, H.; Vilenkin, A.; Yamada, M. CMB spectral distortions from black holes formed by vacuum bubbles. J. Cosmol. Astropart. Phys. 2018, 2018, 059. [CrossRef]

16. Dolgov, A.; Silk, J. Baryon isocurvature fluctuations at small scales and baryonic dark matter. Phys. Rev. D 1993, 47, 4244-4255. [CrossRef]

17. Dolgov, A.D. Massive and supermassive black holes in the contemporary and early Universe and problems in cosmology and astrophysics. Phys.-Uspekhi 2018, 61, 115-132. [CrossRef]

18. Carr, B.; Kohri, K.; Sendouda, Y.; Yokoyama, J. Constraints on Primordial Black Holes. arXiv 2020, arXiv:2002.12778.

19. Hasinger, G. Illuminating the dark ages: Cosmic backgrounds from accretion onto primordial black hole dark matter. arXiv 2020, arXiv:2003.05150.

20. Hawkins, M.R.S. The signature of primordial black holes in the dark matter halos of galaxies. Astron. Astrophys. 2020, 633, A107. [CrossRef]

21. García-Bellido, J. Primordial Black Holes. PoS 2018, 2018, 042. [CrossRef]

22. Clesse, S.; García-Bellido, J. Seven Hints for Primordial Black Hole Dark Matter. Phys. Dark Univ. 2018, 22, 137-146. [CrossRef] 
23. Dokuchaev, V.; Eroshenko, Y.; Rubin, S. Quasars formation around clusters of primordial black holes. Grav. Cosmol. 2005, 11, 99-104.

24. Belotsky, K.; Berkov, A.; Kirillov, A.; Rubin, S. Clusters of black holes as point-like gamma-ray sources. Astropart. Phys. 2011, 35, 28-32. [CrossRef]

25. Toshchenko, K.; Belotsky, K. Studying method of microlensing effect estimation for a cluster of primordial black holes. J. Phys. Conf. Ser. 2019, 1390, 012087. [CrossRef]

(C) 2020 by the authors. Licensee MDPI, Basel, Switzerland. This article is an open access article distributed under the terms and conditions of the Creative Commons Attribution (CC BY) license (http://creativecommons.org/licenses/by/4.0/). 